\title{
Percepciones de profesionales sanitarios sobre medidas no farmacológicas para el manejo del dolor crónico
}

\section{Perceptions of health professionals on non-pharmacological measures for the management of chronic pain}

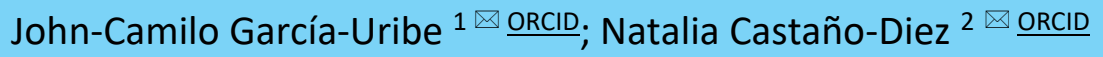

${ }^{1}$ Enfermero profesional, Magister en Bioética. Grupo de investigación Salud Familiar y comunitaria, Facultad de Ciencias de la Salud, Corporación Universitaria Remington, Medellín, Colombia.

2 Enfermera Profesional, Hospital Pablo Tobón Uribe.

Fecha correspondencia:

Recibido: 22 de junio de 2021.

Aceptado: 6 de octubre de 2021.

Forma de citar:

Garcia-Uribe J, Castaño-Diez N. Percepciones de profesionales sanitarios sobre medidas no farmacológicas para el manejo del dolor crónico. Rev CES Enf [Internet]; 2(2):5-27. Disponible en: https://dx.doi.org/10.21615/ cesenferm.6306

\section{Open access}

Licencia creative commons

Ética de publicaciones

Revisión por pares

Gestión por Open Journal System DOI: $10.21615 /$ cesenferm.6306 ISSNe 2745-049X

Publica con nosotros

\section{Resumen}

Introducción: el dolor como problema multicausal, es abordado en gran medida solo desde el ámbito farmacológico. Lo cual, dificulta un manejo integral de los pacientes. Objetivo: identificar las percepciones de un grupo de profesionales de la salud, (profesionales de enfermería y medicina) de la ciudad de Medellín frente al uso de medidas no farmacológicas para el tratamiento del dolor crónico en los adultos. Metodología: investigación cualitativa con enfoque etnográfico particularista. Los participantes fueron seleccionados a través de la técnica de bola de nieve y contactados de manera telefónica para acordar una entrevista presencial en profundidad. Se realizó una transcripción y análisis de información simultáneamente, hasta construir un mapa de categorías. Resultados: cuatro realidades determinan el uso del tratamiento no farmacológico por parte de los profesionales en medicina y enfermería. Estas son: la concepción del dolor que tiene cada profesional de salud, la formación profesional, la experiencia y el ámbito de las instituciones de salud. Conclusión: aunque el manejo no farmacológico pueda ser efectivo existe poca formación de los profesionales en el manejo del dolor y barreras como el contexto social y cultural, la 
occidentalización del conocimiento, burocratización del sistema de salud, protocolización del cuidado, falta de credibilidad en este tipo de tratamientos y dificultades económicas para acceder a este tipo de terapias.

Palabras clave: dolor crónico; investigación cualitativa, investigación en enfermería; etnografía.

\section{Abstract}

Introduction: pain as a multi-causal problem is mainly addressed, only from the pharmacological field. Which, hinders a comprehensive management of patients. Objective: to identify the perceptions of a group of health professionals (nursing and medicine professionals) from Medellín city, regarding the use of non-pharmacological measures for the treatment of chronic pain in adults. Methodology: qualitative research with a particularistic ethnographic approach. The participants were selected through the snowball technique and contacted by telephone to arrange an in-depth face-to-face interview. A transcription and analysis of information was carried out simultaneously, until a category map was built. Results: four realities determine the use of non-pharmacological treatment by professionals in medicine and nursing. These are: the conception of pain that each health professional has, professional training, experience and the scope of health institutions. Conclusion: although nonpharmacological management can be effective, there is little training of professionals in pain management, and barriers such as the social and cultural context, the westernization of knowledge, bureaucratization of the health system, protocolization of care, lack of credibility in this type of treatment and financial difficulties to access this type of therapy.

Keywords: chronic pain; qualitative research; ethnography; nursing research.

\section{Introducción}

El dolor es tanto una sensación como una emoción, la cual, ha estado ligada siempre al ser humano, forma parte de la vida diaria, permitiendo la supervivencia, la búsqueda del placer y dignificar la existencia ${ }^{(1,2)}$. En este sentido, el dolor es definido por la Asociación Internacional para el Estudio del Dolor (IASP) como "una experiencia sensorial y emocional desagradable con daño tisular actual o potencial o descrito en términos de dicho daño" (3).

Es gracias al dolor, que se puede identificar cuando algo va mal, éste es una alerta sobre algún peligro o daño potencial, convirtiéndose en un sistema que ayuda al ser humano a evitar los peligros. Sin embargo, en algunas situaciones, el dolor en sí mismo, puede convertirse en una enfermedad al prolongarse en el tiempo, es decir, aquel dolor crónico que perdura durante 12 
semanas (tres meses) o más ${ }^{(3)}$, dando lugar a un dolor patológico, dejando de ser un síntoma para convertirse en una enfermedad.

El dolor crónico se ha convertido en una problemática mundial, de la cual el ser humano se ha preocupado desde su origen, buscando dar definiciones y tratamientos de múltiples formas a lo largo de la historia; desde concebirlo como un castigo divino y por ende no tratarlo, hasta asumirlo como un daño o un mal funcionamiento del cuerpo y, por consiguiente, solo tratarlo con medicamentos ${ }^{(4)}$.

Las personas que conviven con dolor crónico usualmente presentan una serie de patologías psicológicas concomitantes como: trastornos del sueño e irritabilidad ${ }^{(5)}$; trastorno del apetito, ansiedad y depresión ${ }^{(6)}$. Adicionalmente, el dolor crónico, trae consecuencias socioeconómicas, en primera instancia para el que lo padece, puesto que genera: aislamiento, problemas de pareja, disminución de la libido, disminución de la capacidad para realizar las actividades cotidianas, y un aumento en los gastos secundarios a consultas médicas o tratamientos para el control de este ${ }^{(7)}$. Es entonces, cuando se evidencia al dolor crónico como una enfermedad que, atrae el interés de los profesionales del área de la salud que lo encuentran en su práctica profesional.

Hoy en día se calcula que "en Europa, 138 millones de personas sufren de dolor crónico; en Estados Unidos 100 millones, y en Colombia podrían ser cerca de 14 millones" (8). Debido a esto, 1 de cada 3 individuos con dolor crónico son incapaces o tienen muchas limitaciones para llevar una vida independiente. En el $25 \%$ de los casos, los pacientes consideran que el dolor crónico supone el distanciamiento o la destrucción de las relaciones con familia y amigos. Algunos expertos consideran el dolor crónico como "la epidemia silenciosa" (9).

Respecto a la eficacia del tratamiento no farmacológico en pacientes con dolor crónico, algunos estudios ${ }^{(3,10)}$ han descritos como la relación cuerpo mente tiene un papel vital en el manejo de dolor crónico y como con intervenciones conductuales es posible mediar efectos pequeños a moderados, generalmente a corto plazo, sobre el dolor. Una de las grandes ventajas de las medidas no farmacologías es su potencial como ahorrador de opioides. Algunos autores ${ }^{(11)}$, han descrito este rol que economiza las terapias farmacológicas; entre estas medidas no farmacológicas se encuentran: la terapia de acupuntura, terapia de masajes, manipulación osteopática y quiropráctica, terapias de movimiento meditativo Tai chi y yoga, intervenciones conductuales de mente y cuerpo, componentes dietéticos y estrategias de autocuidado / autoeficacia. 
En varias partes del mundo los profesionales de la salud han comenzado a recomendar a sus pacientes tratamiento no farmacológico para el dolor. Por ejemplo en Chile ${ }^{(12)}$, además de recomendar tratamiento no farmacológico para el dolor, se han preocupado por el mecanismo de acción y la eficacia de este, específicamente de agentes físicos térmicos como: "las compresas húmedas calientes ( $\mathrm{CHC}$ ), la crioterapia, el baño de parafina (BP), la hidroterapia (HD) y los infrarrojos (IR)" (12). Desde la psicología se proponen técnicas no farmacológicas para tratar el dolor, la relajación, la hipnosis, escritura emocional y terapia cognitiva conductual, "las cuales deben hacer parte del tratamiento multimodal interdisciplinario, en el que colaboren conjuntamente médicos, fisioterapeutas, asistentes sociales y psicólogos. A pesar de ello, este tipo de enfoque todavía está poco implantado. En el año 2008, Carlos Barutell, director de la Sociedad Española del Dolor, afirmaba que pocas clínicas del dolor son auténticamente interdisciplinares" (13).

De igual forma, en Colombia, lo más usado en los servicios sanitarios por los profesionales de la salud para el manejo del dolor son las intervenciones farmacológicas, que, aunque son efectivas, no siempre cubren las necesidades de los pacientes, pueden producir efectos colaterales difíciles, crear síndromes de dependencia, son costosas y difíciles de conseguir. Pese a estos inconvenientes del manejo farmacológico, solo al "33\% de los pacientes se les recomienda fisioterapia y solo un 10 \% recurre a otros tratamientos no farmacológicos" (4). Parecen existir factores que limitan la prescripción, recomendación y aceptación de medidas no farmacológicas para el manejo del dolor crónico. Es en este sentido, que se busca explorar las percepciones de dos grupos de profesionales de la salud de la ciudad de Medellín, uno de enfermería y otro de medicina, frente al uso de medidas no farmacológicas para el tratamiento del dolor crónico en los adultos.

\section{Metodología}

Esta investigación fue de tipo exploratorio y de carácter cualitativo con enfoque etnográfico particularista, es decir una etnografía que "se apoya en la convicción de que las tradiciones, roles, valores y normas del ambiente en que se vive se van interiorizando poco a poco y generan regularidades que pueden explicar la conducta individual y grupal en forma adecuada" (14). Y es particularista puesto que para esta investigación se requerirá de pequeñas poblaciones (profesionales de medicina y enfermería) que se encuentren vinculados con la asistencia clínica de pacientes adultos con dolor crónico. Los participantes fueron seleccionados a través de la técnica de bola de nieve y contactados de manera telefónica para acordar una entrevista presencial, cuya duración fue de aproximadamente entre 30 y 40 minutos. 
De esta manera, se entrevistaron 17 profesionales de la salud, de los cuales 10 fueron profesionales en enfermería con especialidades en cuidado al adulto crítico, atención en emergencias y desastres, oncología, rehabilitación, bioética, filosofía, cuidados paliativos, maestría en enfermería y uno de ellos con un doctorado en psicogerontología. Por otro lado, los médicos entrevistados fueron 7 , un médico general y el resto de ellos con especialidades en áreas como: anestesiología, medicina paliativa, dolor, medicina de urgencias, medicina interna, y cirugía general.

Además de la entrevista semiestructurada, se implementaron técnicas de recolección de la información tales como la revisión documental y el diario de campo; teniendo en cuenta los conceptos de dolor, tipo de terapias no farmacológicas, aplicación, contraindicación, beneficios y las barreras para su uso. Para el análisis de la información, simultáneamente se recolectaron los datos, se hizo la transcripción en Microsoft Word, codificación de cada una de las entrevistas, se realizó la matriz de categorías y se dio inicio el proceso de análisis de las entrevistas, el análisis fue manual agrupando y desagrupando los datos tanto de las entrevistas como de los diarios de campo.

La investigación fue desarrollada siguiendo las consideraciones éticas establecidas en los principios del informe Belmont creado el 18 de abril de 1979, y en la Resolución 008430 del 4 octubre de 1993 para el desarrollo de las investigaciones en seres humanos, donde se protegieron los principios de autonomía, respeto, confidencialidad, confianza y justicia. Fue clasificado como un estudio sin riesgo, y aprobado por el Comité de Ética de la Facultad de Enfermería, el 13/07/2015 acta 7.

Durante el contacto preliminar a cada entrevista, en una conversación amena y un proceso deliberativo, se diligenció el formato de consentimiento informado en el que cada participante en pleno uso de sus facultades y sin ningún tipo de presión, aceptó su participación de forma voluntaria en este estudio y la grabación del audio de la entrevista, pudiendo retirarse en cualquier momento. Adicionalmente, al inicio de cada entrevista, se solicitó el consentimiento verbal para dar comienzo a la grabación.

\section{Resultados}

El mapa que se presenta a continuación ilustra las categorías centrales y sus relaciones, lo que permite identificar los ejes más importantes de las percepciones que tienen los profesionales de la salud, de enfermería y medicina, respecto al uso del tratamiento no farmacológico en pacientes con dolor crónico. 


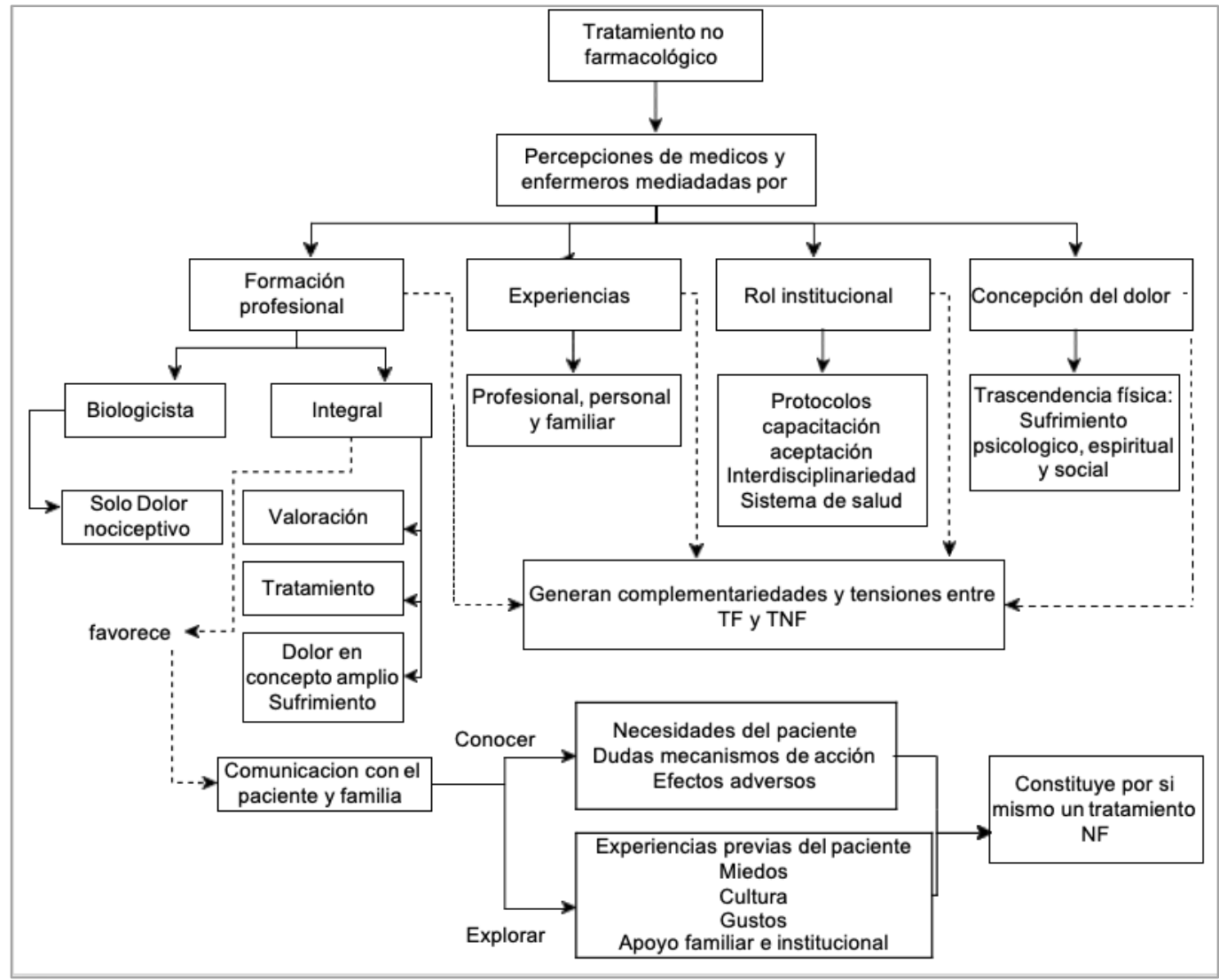

Figura 1. Experiencias y significados referentes al uso de tratamiento no farmacológico. Creación propia.

TF: Tratamiento farmacológico.

TNF: Tratamiento no farmacológico.

En términos generales, hay cuatro realidades que determinan el uso del tratamiento no farmacológico por parte de los profesionales en medicina y enfermería. Estas son: la concepción del dolor que tiene cada profesional de salud, la formación profesional, la experiencia y el ámbito de las instituciones de salud.

A continuación, se presenta cada una de estas categorías, las relaciones que tienen entre sí y las diferencias entre los profesionales de enfermería y los profesionales en medicina.

\section{Formación profesional}

A la luz de esta categoría, fue posible establecer grandes diferencias entre profesionales en medicina y enfermería, así como diferencias al interior de cada uno de estos grupos de profesionales. 


\section{Formación profesional y valoración del paciente con dolor}

En cuanto a la valoración del paciente con dolor, fue posible establecer que es un proceso complejo constituido por los múltiples aspectos que se valoran. De acuerdo con lo referido por los participantes tanto médicos como enfermeros, fue posible establecer que la valoración del paciente con dolor crónico debe incluir características tanto del paciente y su entorno como del dolor mismo.

Tanto profesionales de medicina como de enfermería coinciden que se debe valorar las características del dolor y algunas características del paciente como las físicas. Sin embargo, los enfermeros hicieron más énfasis en valorar aspectos psicológicos y culturales del paciente; mientras que los médicos aspectos físicos y las características del dolor. Con relación a esto, uno de los enfermeros entrevistados refiere:

"Cuando un paciente tiene dolor, a veces le duele más que en la escala de 0 al 10, 12 o 15 o 20, esto es porque el dolor físico también va a acompañado de un dolor en el alma, o porque espiritualmente uno no tiene de dónde hacerse para poder salir adelante en muchas cosas, por eso importante valorar las otras esferas a parte de la física" E1.

Dentro de las características psicológicas del paciente con dolor, algunos profesionales de enfermería y solo el médico con especialidad en dolor y cuidados paliativos, hacen referencia al dolor como sufrimiento durante la entrevista. La cual se presenta a continuación en los siguientes testimonios:

"Muchas veces trabajamos solo con dolor y no con el sufrimiento, entonces yo le quito el dolor físico al paciente, pero el paciente sigue sufriendo por su enfermedad, por su incapacidad o simplemente porque el dolor puede reaparecer, hay que valorar que tanto sufre el paciente, el paciente a veces le duele que no le duela, porque tiene miedo de que le duela y eso lo hace sufrir" E3.

"Lo que pasa es que creo, la cuestión va más allá del dolor, es más evitar el sufrimiento que es una dimensión un poco más global del dolor, va más allá de lo físico, llega a terrenos de lo mental de lo post-sensorial [...] Yo creo que debería haber una cátedra de sufrimiento, manejo humano del sufrimiento, en todas las diferentes carreras que tengan que manejar seres humanos" M1. 
Por su parte, los médicos que centran su atención en la valoración de las características del dolor (tipo de dolor, la duración del dolor, la frecuencia y respuesta al tratamiento farmacológico) consideran que dicho enfoque corresponde en gran parte a la formación profesional basada en el modelo biomédico. Lo cual es resaltado por uno de los médicos entrevistados de la siguiente manera:

"La mayoría de los médicos muchas veces omitimos valorar las otras esferas del paciente, como la social y la psicológica, pero eso es por nuestra formación profesional que está muy centrada en el modelo biomédico y en lo farmacológico" M6.

No obstante, tanto profesionales de enfermería como médicos manifiestan que los conocimientos obtenidos durante el pregrado no son suficientes para dar un manejo integral a los pacientes con dolor crónico. De acuerdo con lo anterior, uno de los entrevistados refiere lo siguiente:

"Uno como médico general no fue formado con las herramientas necesarias para tratar un paciente con dolor crónico, ni farmacológicamente y mucho menos con tratamiento no farmacológico, uno lo remite por decir al internista o neurólogo para que estudie el caso" M5.

De igual forma, los participantes consideran que específicamente en tratamientos no farmacológicos no cuentan con muchos conocimientos por lo cual no pueden brindar un cuidado integral, manifestando que para lograr esto se requiere realizar posgrados en los que el tema del dolor sea trabajado en profundidad. Haciendo alusión a esto, uno de los médicos participante opina lo siguiente:

"Es que el manejo farmacológico y no farmacológico de los pacientes con dolor crónico, tal vez los especialistas del dolor que son los que trabajan en el cuidado paliativo lo tienen claro y algunas personas que trabajan en la parte de psicología y psiquiatría también lo tienen claro, pero curiosamente la gran mayoría de los médicos generales tratantes no tienen muy claro otras medidas adicionales, básicamente porque no se enseña en las facultades de medicina" M1. 
En esta misma línea una enfermera profesional entrevistada manifiesta que:

"En el área clínica no se hace, porque no se conoce bien cómo valorar y tratar un paciente con dolor, entonces se centran solo en el tratamiento farmacológico y el tratamiento farmacológico sin valorar efectivamente no sirve, porque la valoración solo se basa en la escala análoga del dolor, no se exploran otras dimensiones del dolor humano, simplemente nos quedamos en la intensidad del dolor, y eso que a veces ni conocen las escalas del dolor" M2.

En lo referente a la inclusión de las medidas no farmacológicas para el tratamiento del dolor crónico en el pensum académico y la participación de las instituciones educativas, se encontró que existen falencias en cuanto la forma de enseñarlas y su modo de implementación, dado que algunos refieren que hace falta investigaciones que demuestren su eficacia en el tratamiento del dolor o por ser en su mayoría provenientes de otro modelo médico de atención diferentes del occidental, respecto a esto, dos participantes afirman lo siguiente:

"Hace falta más tiempo para poder dedicar a un área como es el dolor, hace falta un poquito más de tiempo para explicarles y enseñarles mejor a los estudiantes cómo se implementan y hay que posibilitar que los estudiantes exploren esta área" EM1.

"Nosotros casi no trabajamos el tema del dolor sino cosas muy puntuales y en unos momentos muy cortos... dentro de la misma formación, dentro del currículo de enfermería. Por ejemplo, en el sexto semestre trabajamos dolor oncológico, manejo del dolor; en el séptimo manejan todo muy centrado en el dolor posquirúrgico, pero nos quedamos muy cortos porque son 6, 8 horas o 10 para todo un currículo, es demasiado corto" E5.

\section{Experiencia del profesional}

Algunas profesionales de acuerdo con las experiencias vividas con pacientes ponen de manifiesto la relevancia que tiene el proporcionar un ambiente tranquilo y adecuado para mejorar el estado de salud de las personas que padecen dolor; puesto que el ruido puede exacerbar en algunos pacientes el dolor y dificultar el control de este. Paradójicamente esto fue reportado por profesionales de enfermería que se encontraban como pacientes en servicios de hospitalización, entendido como una paradoja en el sentido de que estos servicios deben caracterizarse por ser tranquilos para facilitar el descanso y reposo de los pacientes. El testimonio de la enfermera frente a esta situación es el siguiente: 
"Cuando yo estaba hospitalizada escuchaba las conversaciones en voz muy alta de la enfermera, escuchaba qué tenían los otros pacientes, pues hablaban de eso continuamente, además me tocaba escuchar reggaetón en temporada decembrina, uno con un dolor bien insoportable y con bastante ruido se estresa más, entonces todo el día estaba con dolor y ni los medicamentos me servían" E3.

De igual forma varias enfermeras mencionan la importancia que tiene experiencias del profesional a la hora de valorar el paciente con dolor, esto permite una relación empática y abre la puerta a tratamientos no farmacológicos En relación con el tema se presentan dos testimonios de profesionales de enfermería, en donde ellos afirman lo siguiente:

"Cuando yo estaba hospitalizada con mi dolor Neuropático de muchos años, las enfermeras y los médicos decían "dolor, dolor", y listo creen que no es sino administrar el tratamiento fármaco para mejorar el dolor y no piensan en las consecuencias y si no miren que yo soy diabética, producto de tratarme ese dolor crónico con esteroides, yo por eso trato de hablarles de otras cosas no solo de medicamentos" E3.

"yo tuve una lesión hace 7 meses en la mano y los analgésicos generalmente me funcionaron muy poco. Me funciona mucho más la terapia y los medios físicos, una cosa que conocí que se llama kinesiotaping que no conocía y que conocí ahora pues por lo de mi lesión, que funcionan mucho mejor que los analgésicos que la verdad funcionan como ahí, pero a veces tiene más efectos negativos y no funcionan" E5.

Ciertas enfermeras también manifiestan que muchos de los aprendizajes empíricos en sus hogares les ha servido para manejar el dolor crónico en los pacientes, resaltando que estas medidas son más eficaces en el dolor crónico no maligno que en el maligno, ya que éste requiere una complejidad y uso conjuntos de las medidas farmacológicas y no farmacológicas, y esto se puede evidenciar con el siguiente testimonio:

"Y nos ha funcionado, nos han funcionado las bebidas que nos enseñaron nuestras mamás de Canela, de Manzanilla, de Caléndula pues obviamente no para dolores crónicos, de tipo no maligno, porque ya cuando el dolor es maligno, es muy difícil" E4.

Dado que la naturaleza de los seres humanos es buscar los métodos necesarios para intervenir todo lo que acontece en la vida diaria, en el caso particular del uso de estas alternativas para el tratamiento del dolor crónico por parte del personal de salud, se percibe que su 
implementación está altamente influenciado por las experiencias personales y familiares de quienes padecen dolor y de los profesionales de la salud, por ello, la importancia de la participación de la familia, dado que son quienes aportan mayor información de los métodos culturales para tratar el dolor, enriqueciendo así, el conocimiento profesional:

"El conocimiento digamos empírico si, (silencio prolongado) a un paciente terminal en casa la familia le hacía cositas de estas, masajes, compresas, ejercicios, ponerle música, pues como lo que podían hacer con ella y al fin no sabemos cuál de todas la calmaba, si los medicamentos de uno o lo de ellos, pero a todos nos daba la sensación de que eso ayudaba y que lo debíamos hacer y de estar acompañándola, y de estar dedicando el tiempo que merecía" E6.

De acuerdo con todo lo anterior, se puede entender que todas las experiencias personales y familiares brindan herramientas importantes a los profesionales de la salud a la hora de intervenir pacientes con dolor crónico. Aclarando que para la implementación de las medidas no farmacológicas se requiere tanto de las percepciones y experiencias previas de las personas que padecen dolor, como de soportes científicos o alguna evidencia que garanticen su eficacia en el tratamiento del dolor crónico; de tal manera que los profesionales puedan aplicarlos en su vida y ejercicio profesional.

\section{Rol institucional}

En cuanto al rol de las instituciones administrativas y del sistema de salud con el que se cuenta actualmente, los participantes hacen especial referencia a temas como la no inclusión de las medidas no farmacológicas en el Plan Obligatorio de Salud (POS), la diversidad en los trámites, los pocos profesionales médicos especialistas en dolor y la negativa ante la autorización para citas, medicamentos y tratamientos. Por lo tanto, la principal falencia que se presenta al incluir las medidas no farmacológicas en el tratamiento del dolor crónico y realizar un seguimiento continuo a éste, es porque la dinámica en los servicios de salud está encaminada en realizar los procedimientos que se encuentren en el Plan Obligatorio de Salud para evitar los incrementos en los costos y sus diligencias. Teniendo en cuenta lo anteriormente mencionado, un participante manifiestan que:

"Las consecuciones de las citas son muy difíciles, por muchas trabas administrativas, trabas en cuanto a las autorizaciones para determinadas especialidades, eso hace que un paciente tenga una cita en 6 meses con un especialista, todo ese tiempo con dolor mal controlado" E5. 
Algunos participantes también hacen referencia a los limitantes que se establecen en las instituciones de salud debido a la existencia de protocolos, ya que por estos muchos de los profesionales de la salud dejan de realizar procedimientos importantes para el tratamiento del dolor crónico solo porque en el protocolo no está escrito, así lo refieren claramente dos de los participantes:

"Hoy en día se habla en términos de protocolo. Que está en el protocolo, que está en la política, entonces la gente se va ciñendo a todo eso porque por aquí es que hay que seguir, esta es la guía, no todo mundo cabe ahí, pero la mayoría si, entonces si eso está estandarizado, entonces se hace si no, no" E9.

"es difícil el acceso a muchos servicios de salud y no sé si ustedes saben cuánto se puede demorar para que se autorice un medicamento no POS, en una EPS buena se puede demorar alrededor de 15 días" M5.

Cuando se hace referencia a la autorización de las citas y a los diversos procesos que se deben realizar para que se dé la continuidad de un tratamiento, algunos enfermeros y médicos coinciden en que para poder conseguir la atención adecuada se necesitan múltiples trámites que van a tener como resultado del proceso la no autorización para recibir la atención. Por lo que el mismo sistema y las instituciones son una barrera para el control del dolor y en algunos casos para la implementación de medidas no farmacológicas.

\section{La concepción del dolor}

Esta categoría permite conocer el significado del dolor para los profesionales de salud. De acuerdo a las diversas concepciones de los profesionales acerca del dolor y la vida del paciente que lo padece, radica la forma de abordar el dolor; es decir, si el profesional de salud concibe el dolor de una manera holística, como un síndrome que afecta todas las dimensiones del ser humano, será abordado más ampliamente, por lo que estaría no solamente abierto al uso de tratamientos no farmacológicos, sino que también se interesará por conocer un poco más acerca de ellos para posteriormente recomendarlos. Por el contrario, si el profesional considera el dolor como un problema netamente físico, solo emplea tratamientos cuyo objetivo sea intervenir la esfera física como es el caso del uso de los analgésicos. De acuerdo con lo antes mencionado, uno de los participantes afirma:

"El dolor es más complejo que la simple manifestación neurológica o la simple manifestación o la expresión somática del dolor... aunque hay muchas herramientas, se 
necesita el concurso de muchas personas, de muchos especialistas, apoyo familiar... el hecho de no poder desarrollar su actividades que interfieren con el sustento, con la dinámica familiar, generan conflictos, peleas de parejas, cosas que afectan y atraviesan todas las esferas de la vida de los pacientes de una manera muy severa, muy impactante, muy catastrófica en algunos casos, algunas personas llegan a tomar decisiones autolesivas, intentos suicidas, pues son situaciones bastante complejas, por lo tanto la valoración y el manejo del dolor debe valorar todos esos aspectos" M2.

Para dar una explicación fisiológica al proceso del dolor, algunos enfermeros hacen alusión a la teoría de las compuertas del dolor y afirman lo siguiente:

"Cuando uno tiene una sensación dolorosa, la fibra nerviosa se acoge a la médula espinal para llevar esa sensación dolorosa hasta el encéfalo y se devuelve para que se manifieste el dolor que tiene, pero en esos trayectos puede haber moduladores del dolor, porque por la misma vía del dolor se transmite otras sensaciones, esas otras sensaciones pueden ser caricias, masajes, por eso a veces sirven" E2.

Los participantes mencionan que la valoración del dolor se debe hacer siempre de manera individualizada y sin generalizaciones; aunque el dolor tenga manifestaciones físicas evaluables de manera objetiva, es la dimensión subjetiva del dolor la más compleja e importante. Por ello, se debe tomar al paciente como un interlocutor válido, creyéndole cuando afirma que tiene un dolor de determinadas características y sin establecer juicios de valor. Por consiguiente, varios testimonios expresan lo siguiente:

"Se debe creer al paciente cuando dice que tiene dolor, hay que creerle y lamentablemente muchas veces no se les escucha o por el contrario se les tilda que son cansones," E1.

"Los profesionales que tienen tanta experiencia con pacientes que padecen dolor crónico, pierden esa sensibilidad ante el sufrimiento humano, no les creen a los pacientes piensan que se están haciendo, que quieren incapacidades y ya o que se volvieron adictos a la morfina y como no les creen pues no hacen nada" E10.

"Los profesionales de enfermería no pueden medir la intensidad del dolor que tiene el paciente, mucho menos pueden decir que es un dolor falso, porque el dolor es una percepción propia y no es lo mismo lo que el profesional sienta a lo que sienta el otro" E2. 
"Si no le creemos al paciente entonces no utilizamos lo que tenemos a la mano, como abogar por el paciente ante el médico para solicitar más analgésicos, evaluar la efectividad de los medicamentos, utilizar tratamientos no farmacológicos sencillos como la distracción; y pues si no hacemos lo que está a nuestro alcance no vamos más allá como sugerir interconsulta por especialistas o realizar tratamientos no farmacológicos que requieran mucho tiempo" E9.

Incluso en aquellos casos en que el profesional de la salud identifique que el dolor no tiene un origen netamente físico, se le debe prestar atención al paciente porque finalmente está somatizando un dolor que puede ser de origen psicológico, psíquico o cognitivo. De acuerdo con esto, uno de los participantes afirma:

"El dolor también es un llamado, un llamado comunicativo de atención de que algo está pasando, de que el paciente está solo, está triste. Entonces los pacientes muchas veces que hacen, somatizan, llevan al nivel físico las alteraciones que tienen del nivel psíquico, cognitivo, mental, ahí empiezan con dolores, es una forma de llamar la atención. El dolor y la enfermedad son formas de conocer la finitud y las limitaciones que como seres humanos tenemos, pero también son formas de hacer llamados de atención y muchas veces lo que se quiere decir es, acompáñeme, sóbeme, búsqueme, venga no me deje solo, venga asístame, venga que estoy solo, venga que tengo miedo, más que el dolor físico, más que la herida, más que la cosita que tenga es un llamado a que algo está funcionando mal y que algo está pasando" E6.

\section{Las tensiones entre el tratamiento farmacológico y el no farmacológico}

Es importante mencionar que, en cuanto a las barreras encontradas durante el proceso de recolección y análisis de la información, las causas más comunes para el rechazo de las medidas no farmacológicas en el tratamiento del dolor, son la falta de credibilidad que les tienen debido a que algunas personas y sus familiares consideran que solo las medidas farmacológicas son efectivas dado que sus resultados son más inmediatos en comparación con las medidas no farmacológicas.

"Algunos no creen, son escépticos entonces no las profesan, no las creen, no las impulsan. Cuando creamos de forma ferviente, cuando los recursos para implementarlas estén disponibles será mucho más factible implementarlas" E2.

Algunos participantes reconocen que este tipo de tratamiento no cuenta con suficiente evidencia científica que respalde su eficacia, de manera que pueda aumentar el índice de uso 
por los profesionales y así mismo en las instituciones de salud. En relación con esto, uno de los participantes infiere lo siguiente:

"A través de un método científico, esperamos que una terapia que mejore sea reproducible y que sea aplicable a la mayoría de las personas, y el caso del dolor como es una experiencia que atraviesa la subjetividad, hay muchas cosas que no se pueden estandarizar, entonces se vuelve bastante complejo la forma de demostrar que una estrategia sea tan efectiva como para que la conducta médica cambie de forma radical" M2.

Sin embargo, el desconocimiento es un generador de discrepancias:

"Hay muchos tipos de terapias que pueden ser muy buenos coadyuvantes, pero por el desconocimiento básicamente no se usan y podrían ser muy eficaces, imagínese que todavía hay enfermeras que piensan que el cuidado paliativo es solo para los pacientes con cáncer o que es solo para los pacientes que se van a morir, no tienen una mirada integral del dolor".

\section{Discusión}

En términos generales, se encuentra que tanto profesionales de enfermería como de medicina, concuerdan en que el dolor crónico es un problema multidimensional y de difícil manejo, dado que se presentan falencias en la atención de las personas que padecen dolor, en la utilización de las escalas para la valoración del dolor y en el uso de las medidas farmacológicas y no farmacológicas, por lo que en muchos casos las personas que padecen de dolor crónico deben recurrir repetitivamente a varios profesionales y a múltiples tratamientos.

En este sentido, Serrano ${ }^{(15)}$ afirma que "el dolor es una experiencia personal privada", aunque exista empatía y compasión la posibilidad de sentir lo que otra persona siente es imposible, la posibilidad de la cercanía permite una mejor comprensión de la dimensión del dolor, pero nunca con una precisión exacta. La valoración del dolor es un proceso inmerso en la relación profesional de salud - paciente, como proceso relacional, está inmerso en la subjetividad de los sujetos. Esto supone que, incluso utilizando métodos objetivos como escalas y guías de valoración, los resultados estarían siempre condicionados al contexto y diversos factores individuales ligados a las experiencias previas, creencias, significados, cultura, educación y nivel socioeconómico. En tal sentido, es necesario reconocer las escalas y guías de valoración, como 
herramientas que facilitan el proceso de valoración y tratamiento, pero incapaces de contener el holismo necesario para el manejo del dolor crónico.

De acuerdo con los resultados, la dimensión del sufrimiento es uno de los aspectos fundamentales para dar un enfoque integral, en este mismo sentido, la escuela fenomenológica de psiquiatría afirma que "el dolor más que una sensación o una percepción, es un sentimiento cualitativamente displacentero, que da lugar a un sufrimiento, de lo cual deriva la profundidad psicológica con que es vivido por el sujeto adolorido" (16)

No obstante, existen barreras que limitan el alivio del dolor crónico y por ende el alivio del sufrimiento humano ${ }^{(17)}$. Estas barreras, son tanto a nivel institucional como a nivel profesional. A nivel institucional, es importante mencionar que desde la estructura y enfoque del sistema general de salud existen obstáculos. Adicionalmente, existen factores de los profesionales como la falta de sensibilidad y el desconocimiento de todos los métodos para el tratamiento del dolor crónico. En esto coinciden tanto profesionales en medicina como de enfermería, participantes de la investigación.

Este desconocimiento frente al tema ya ha sido planteado por otras investigaciones ${ }^{(18)}$, dado que muchas veces el abordaje del dolor se ve limitado, porque los profesionales de la salud, en especial los enfermeros, desconocen la configuración fisiológica del dolor, los mecanismos de acción de la farmacoterapia y la existencia de herramientas disciplinares que permiten una valoración integral y proporcionan elementos para orientar el manejo multidimensional e integral del paciente con dolor crónico. De igual forma los estudiantes en formación y los recién egresados, no tienen los conocimientos necesarios para enfrentar un problema de tal magnitud como lo es el dolor crónico ${ }^{(18,19)}$, por lo tanto, el ciclo desconocimiento - apatía - incredulidad mirada reduccionista del paciente con dolor, tiende a perpetuarse y con ello los pacientes siguen sufriendo a causa del dolor. No obstante, hay una luz en el camino para sobrepasar estas limitaciones y tener la oportunidad de brindarle un tratamiento integral a todas las personas que sufren de dolor, se requiere que todos los profesionales amplíen el panorama frente a la concepción reduccionista que se tiene acerca del dolor.

Por ejemplo, considerar, en el sentido de Max Scheler (20), el dolor como sensación vital, que reside en la esencia de lo humano, es tan humano que, siendo una característica común de especie, adquiere rasgos propios dependientes del sujeto, al momento y el lugar. El dolor como vivencia, como experiencia y como historia, da cuenta de la existencia del sujeto, adolorido. El dolor como sensación remite a la consciencia de vida, pero a su vez, a la consciencia de 
vulnerabilidad e interdependencia. Es allí, desde la vulnerabilidad y la contingencia que, el dolor se convierte en un recordatorio, en una remembranza, en un cuerpo que grita y llama en busca de la anestesia placentera de la compañía y el encuentro con el otro, porque la relación dicotómica dolor y placer da significado a la existencia de los sujetos, el placer adquiere sentido, cuando se ha padecido el dolor y paradójicamente el dolor adquiere significado cuando se ha conocido el placer.

Por otro lado, estos profesionales difieren sustancialmente en las repercusiones que trae el dolor crónico, la manera de abordarlo y los diferentes tratamientos instaurados para el control de este. Aclarando que estas diferencias se hacen más evidentes al momento de implementar los tratamientos no farmacológicos, ya que estos tratamientos no hacen parte de la formación académica básica profesional; están relacionados con formación en posgrados de dolor y cuidados paliativos, además, se relacionan directamente con prácticas culturales y experiencias aprendidas de otras disciplinas como la medicina oriental, el yoga, la psicología, y otras alternativas terapéuticas.

Sin embargo, actualmente se ha dejado un poco de lado los escepticismos en relación con el uso de las medidas no farmacológicas debido a que, tanto médicos como profesionales de enfermería las implementan cada vez más en pacientes que padecen dolor crónico ${ }^{(21)}$. Aunque difieren considerablemente sobre el enfoque que les dan a las medidas no farmacológicas, dado que los médicos centran más su atención en las características del dolor y en el abordaje desde la parte física; un ejemplo claro de esto es el uso de medidas invasivas como los bloqueos, las rizotomías, neurólisis quirúrgica y química, tracciones esqueléticas, entre otras más que requieren de una intervención quirúrgica o corpórea.

Por otro lado, los profesionales de enfermería centran su atención no solo en las características del dolor sino también en las características del paciente y es allí donde se crea las diferencias más notables en el uso de las medidas no farmacológicas porque además de realizar intervenciones físicas, este tipo de profesionales abordan la parte psicológica, espiritual y el sufrimiento de los pacientes. Implementando así, las medidas no farmacológicas como la exploración de los miedos y temores, la escucha, el acompañamiento constante, la participación de la familia, el promover el descanso y el confort de las personas que padecen dolor, el mitigar el sufrimiento mediante la resolución de inquietudes y el fortalecimiento de la $\mathrm{fe}$, tratando el dolor teniendo en cuenta las costumbres culturales, y en casos de que los pacientes estén en el final de su vida, los profesionales de enfermería permiten que estas personas se preparen para la muerte ${ }^{(22)}$. Así se logra que los pacientes puedan solucionar 
situaciones, asuntos pendientes, disgustos o conflictos que no les permiten tener tranquilidad y un espacio de armonía en su dolor.

La escucha como cuidado de enfermería, más que una intervención, es una necesidad para la práctica y para vida. "La necesidad de ser escuchado y atendido por el otro se hace presente desde el momento mismo del nacimiento. La primera palabra es un grito. A partir de allí podría elaborarse una semiología de la queja que diga mucho acerca del dolor y el sufrimiento humano. La primera conclusión sería que hablar cura y que la represión del acto simbólico incrementa la tensión dolorosa" (15). El enfermero a veces se queda apartado, con los brazos cruzados esperando seguir un protocolo o una orden médica, a pesar de que, en las intervenciones NIC ${ }^{(23)}$, existen aproximadamente 40 intervenciones para el manejo del dolor crónico y que incluso dentro de las relacionadas con la administración de medicamentos se encuentran implícitas actividades no farmacológicas. Intervenciones un tanto olvidadas como Contacto (NIC 5460), Distracción (NIC 5900) y Escucha activa (NIC 4920), deben desempolvarse y aplicarse en pacientes con dolor crónico, incluso no solo por enfermería sino por todos los profesionales involucrados en la atención del paciente.

\section{Conclusiones}

Los profesionales de la salud entrevistados enfatizan en la poca formación que se da en las universidades e instituciones de educación superior sobre el dolor crónico y el tratamiento que debe dársele a este. Este aspecto presenta notables problemas en la forma cómo los profesionales afrontan el dolor de los pacientes, eligen los tratamientos más eficaces para estos, vinculan a las familias de los pacientes a su tratamiento, etc. A su vez, esta falta de formación puede incidir en la dificultad para diferenciar entre los aspectos objetivos del dolor y la experiencia subjetiva que puede manifestar el paciente como una experiencia de sufrimiento. Además, es indiscutible la necesidad de realizar investigaciones en el medio local y contextualizadas de modo que pueda darse mayor visibilidad de la eficacia de las medidas no farmacológicas en el tratamiento del dolor crónico, de tal manera que esto pueda incidir en los programas de formación y en las mismas prácticas de salud.

Los sistemas de salud, y diversas entidades prestadoras de salud, en particular, inciden en el poco uso que se hace de medidas no farmacológicas para el tratamiento del dolor. Esto se puede evidenciar en lo señalado por los entrevistados: a) burocratización del sistema de salud, b) protocolización del cuidado, c) falta de credibilidad en este tipo de tratamientos y en la mal llamada medicina alternativa, d) dificultades económicas para acceder a este tipo de tratamientos, entre otras. Esto conduce a reflexiones sobre las políticas de salud que inciden 
en el sesgo de los profesionales y los pacientes sobre el uso exclusivo de tratamiento farmacológico.

Algunos de los profesionales de salud entrevistados señalan que muchos de sus conocimientos sobre el uso de medidas de tratamiento no farmacológico están sustentados en la propia experiencia de enfermedad o la de uno de sus familiares o simplemente gracias a una especialización en el tema. Este aspecto permite reflexionar sobre la falta de educación para profesionales y pacientes, sobre la base de la evidencia científica, en el uso de este tipo de medidas. En el caso de los pacientes se deben plantear propuestas de educación en salud que conduzcan a evitar posibles empeoramientos y/o dificultades de su condición de dolor. Esto a propósito de los casos en los cuales los pacientes usan tratamientos prescritos por personal no idóneo ni competente en el tema.

Se requiere de una concepción holística del dolor, para dar un manejo integral a todos los pacientes que sufren por esta causa, para que como profesionales de salud se pueda abordar el dolor con todos los componentes físicos, culturales, espirituales y psicológicos. De esta manera, los profesionales tendrán más herramientas para realizar una valoración integral al paciente con dolor crónico y por consiguiente identificar tratamientos específicos para cada uno de los hallazgos encontrados en ésta. Lo anterior exige realizar un análisis detallado sobre las repercusiones del dolor en la calidad de vida de ese paciente, su familia, el ámbito laboral y social. El reconocimiento y comprensión del papel que cumple el dolor crónico en la vida del paciente puede humanizar y ampliar el uso de tratamientos no farmacológicos para este tipo de casos.

De acuerdo con los participantes de este estudio, se pueden identificar problemas relacionados con la formación académica, que centra su atención en la medicalización de la atención y cuidado del paciente con dolor. Sin embargo, es un problema que trasciende la formación académica, puesto que, en esencia, parte de una concepción reduccionista-biologicista del dolor, sin dimensionar la parte psicosocial. La dimensión holística del paciente con dolor crónico debe partir de una comprensión fenomenológica del problema que trascienda las barreras epistemológicas biológicas y fisiológicas. Se requiere explorar lo que significa para un paciente vivir con dolor crónico, las implicaciones para su círculo social, e incluso explorar causas subyacentes inexploradas. El cuerpo que grita y los labios que callan yacen, en muchas ocasiones, frente a un profesional de salud ciego, sordo y mudo, automatizado y acostumbrado a la farmacologización de la enfermedad e incluso de la vida misma. 
El paciente con dolor crónico no puede ser estricta preocupación de médicos y enfermeras especialistas. El dolor como componente ontológico debe involucrar todos los ámbitos del cuidado. Aunque no fue el foco de este estudio, es posible pensar en diagnósticos e intervenciones propias de la disciplina orientadas al cuidado de este tipo de pacientes, por ejemplo:

Es necesario el desarrollo de más investigaciones relacionadas con el uso del tratamiento no farmacológico con otros enfoques, como por ejemplo, su implementación en dolor agudo, validación de instrumentos que contemplen la dimensión del sufrimiento, la participación de los pacientes para la recolección de la información, que permita conocer o abordar de manera específica la efectividad y aplicación de este tipo de medidas desde su experiencia personal y por parte de los profesionales de salud. También, incluir otro tipo de pacientes como son los pediátricos y profesionales que estén en contra de este tipo de tratamiento, de tal forma que sea posible realizar una discusión más amplia acerca del por qué, pese a su efectividad, continúan muchos profesionales en desacuerdo con su aplicación.

Existen diferencias entre la mirada de profesionales en medicina y enfermería que parecen subyacer en la formación y el enfoque profesional. La mirada del cuidado de enfermería parece ser en primera instancia más integral, en ambas profesiones el enfoque se torna más integral entre lo farmacológico y no farmacológico cuando ahondan en el cuidado y manejo de pacientes con dolor, cuando la práctica se los demanda o cuando realizan estudios de postgrado en dolor y cuidados paliativos. Sin embargo, estos límites para el cuidado y atención de pacientes con dolor deben trascenderse, las estructuras cognitivas de los profesionales deben abrirse a explorar otras terapias y a resistir ante la medicalización del y de la vida cotidiana.

\section{Conflictos de interés}

No se presentaron conflictos de interés.

\section{Referencias}

1. Araújo M, Scheffer V, Viera C. Pain as the fifth vital sign: nurse's practices and challenges in a neonatal intensive unit care. Brazilian Journal Of Pain [Internet]. 2019 [citado 8 de noviembre de 2021]. Disponible en: https://www.scielo.br/scielo.php?script=sci arttext\&pid=S259531922019000300232\&lng=en\&nrm=iso\&tlng=en 
2. Benavides C, Montoya C, Nel J. El dolor, quinto signo vital. Fascicul08. 2010; 1:02-7.

3. Treede RD, Rief W, Barke A, Aziz Q, Bennett MI, Benoliel R, et al. Chronic pain as a symptom or a disease: the IASP Classification of Chronic Pain for the International Classification of Diseases (ICD-11). PAIN. 2019;160(1):19-27. [citado 10 de octubre de 2021]. DOI: 10.1097/j.pain.0000000000001365

4. Kopf A, Patel N, editores. Guía para el manejo del Dolor en condiciones de bajos recursos. [Internet]. Washington, D.C: International Association for the Study of Pain; 2010 [citado 22 de enero de 2021]. 412 p. Disponible en: https://s3.amazonaws.com/rdcmsiasp/files/production/public/Content/ContentFolders/Publications2/FreeBooks/Guide toPainManagement Spanish.pdf

5. Mencías $A B$, Rodríguez JL. Trastornos del sueño en el paciente con dolor crónico. Rev Soc Esp Dolor. [Internet]. Diciembre de 2012;19(6):332-4. [citado 10 de octubre de 2021]. Disponible en: https://scielo.isciii.es/pdf/dolor/v19n6/revision4.pdf

6. Arango CA, Rincón HG. Trastorno depresivo, trastorno de ansiedad y dolor crónico: múltiples manifestaciones de un núcleo fisiopatológico y clínico común. Rev Colomb Psiquiatr. [Internet]. 2018;47(1):46-55. [citado 22 de enero de 2021]. Disponible en: http://www.scielo.org.co/scielo.php?pid=S003474502018000100046\&script=sci abstract\&tlng=es

7. Failde I. El dolor crónico, algo más que un problema de quien lo padece. Rev Soc Esp del Dolor. [Internet] 2014;21(1):2. DOI: 10.4321/S1134-80462014000100001 Disponible en: https://scielo.isciii.es/scielo.php?script=sci arttext\&pid=S1134$\underline{80462014000100001}$

8. Guerreiro M, Gómez MP. Estudio nacional sobre prevalencia, características e incapacidad asociada al dolor en la población colombiana [Internet]. Dolor. 2019;8(1):17-26. [citado 8 de Diciembre de 2021]. Disponible en: https://dolor.org.co/biblioteca/revistas/Revista\%208 1.pdf\#page=17

9. Moscoso J. El dolor como epidemia [Internet]. Pasaj Rev Pensam Contemp. 2015;48:308. [citado 22 de enero de 2021]. Disponible en: http://digital.csic.es/handle/10261/166554 
10. Chou R, Deyo R, Friedly J, Skelly A, Hashimoto R, Weimer M, et al. Nonpharmacologic Therapies for Low Back Pain: A Systematic Review for an American College of Physicians Clinical Practice Guideline [Internet]. Ann Intern Med. 2017;166(7):493-505. [citado 22 de enero de 2021]. Disponible en: https://www.acpjournals.org/doi/10.7326/m162459. DOI: $10.7326 / \mathrm{m} 16-2459$

11. Tick H, Nielsen A, Pelletier KR, Bonakdar R, Simmons S, Glick R, et al. Evidence-Based Nonpharmacologic Strategies for Comprehensive Pain Care: The Consortium Pain Task Force White Paper [Internet]. 2018;14(3):177-211 [citado 20 de enero de 2021]. DOI: 10.1016/j.explore.2018.02.001

12. Pavez FJ. Agentes físicos superficiales y dolor: Análisis de su eficacia a la luz de la evidencia científica. Rev Soc Esp Dolor. 2009;16(3):182-9.

13. Moix I. Terapias Psicológicas para el Tratamiento del Dolor Crónico. Clínica Salud. marzo de 2011;22(1):41-50.

14. Martínez Miguel. La investigación cualitativa etnográfica en educación: manual teóricopráctico. México: Trillas; 1998.

15. Serrano A. Nuevo Ensayo en Fenomenología del Dolor. Ápeiron Estudios de Filosofía. 2015; 3: 129-135.

16. Malpica CR, Díaz JE, Esser MR. Complejidad del dolor y el sufrimiento humano. Rev Latinoam Psicopatol Fundam. 2004;7(3):70-81.

17. Zaki J, Wager TD, Singer T, Keysers C, Gazzola V. The Anatomy of Suffering: Understanding the Relationship between Nociceptive and Empathic Pain. Trends Cogn Sci. 2016;20(4):249-59.

18. Achury DM. Dolor: la verdadera realidad. Aquichan. 2008;8(2):13.

19. Zavala M, Fernanda G. Nursing Students' Knowledge and Management of Pain. Aquichan. 2008;8(2):212-26.

20. Ramirez M. La propuesta de Max Scheler como resolución ante el problema de la ininteligibilidad del dolor. STOA Revista del Instituto de Filosofía de la Universidad Veracruzana. 2020;11(21): 82-97. 
21. Castro H, Jairo J. La mal llamada medicina alternativa. Rev Cienc Salud. 2017;15(1):5-6.

22. Achury D. Aplicación de la teoria de los sintomas desagradables en el manejo del dolor. Investig En Enferm Imagen Desarro. 2007;9(1):7-18.

23. McCloskey JC, Bulechek GM. Clasificación de intervenciones en enfermería (NIC), cuarta edición. Madrid: Elsevier; 2005. 